\title{
Deletion of C9orf53 promotes the growth of head and neck squamous cell carcinoma and is associated with poor prognosis of patients with head and neck squamous cell carcinoma
}

\author{
GUO DONG WANG ${ }^{1}$, JIAN TAO HUANG ${ }^{1}$, YUAN LIU $^{1}$, YANG WU ${ }^{1}$, \\ XIAO QING CHEN ${ }^{1}$, YUN FU ZHAO ${ }^{1}$ and DA LIN WANG ${ }^{2}$ \\ ${ }^{1}$ Department of Stomatology, Changzheng Hospital, Second Military Medical University, Shanghai 200003; \\ ${ }^{2}$ Department of Stomatology, Changhai Hospital, Second Military Medical University, Shanghai 200438, P.R. China
}

Received September 24, 2015; Accepted May 31, 2018

DOI: $10.3892 / \mathrm{ol} .2018 .9675$

\begin{abstract}
Head and neck squamous cell carcinoma (HNSCC) is the fifth most common carcinoma worldwide, and accounts for $\sim 600,000$ new cases every year. The information on the molecular carcinogenesis of HNSCC is very limited. In the present study, the role of C9orf53 in HNSCC was investigated. The levels of C9orf53 were assayed by reverse transcription-quantitative polymerase chain reaction. The levels of C9orf53 in cells were overexpressed by overexpression plasmid and inhibited by small-interfering RNA. Cell proliferation was assayed by MTT, and cell apoptosis was assessed by FACS analysis. It was demonstrated that C9orf53 deletion was associated with a decreased survival of patients. The level of C9orf53 in HNSCC tissues was lower compared with the matched normal tissues adjacent to tumors. A lower expression of C9orf53 promoted cell proliferation, and the overexpression of C9orf53 induced cell apoptosis. In conclusion, a low level of C9orf53 in HNSCC promoted the growth of HNSCC cells, which might be associated with the low survival rate of patients with HNSCC.
\end{abstract}

\section{Introduction}

Head and neck squamous cell carcinoma (HNSCC) encompasses a heterogeneous group of tumors with aggressive nature and is the fifth most common carcinoma worldwide $(1,2)$.

Correspondence to: Dr Yun Fu Zhao, Department of Stomatology, Changzheng Hospital, Second Military Medical University, 415 Fengyang Road, Shanghai 200003, P.R. China

E-mail: zhaoyf1818@126.com

Dr Da Lin Wang, Department of Stomatology, Changhai Hospital, Second Military Medical University, 168 Changhai Road, Shanghai 200438, P.R. China

E-mail: wang_dento@163.com

Key words: CDKN2A antisense RNA 1, head and neck squamous cell carcinoma, cells growth, patient survival
HNSCC accounts for $4 \%$ of all malignancies worldwide and $5 \%$ of all cancer mortalities (3).

The choice of cancer treatment depends on the site of the primary tumor, the stage of the disease, treatment toxicities and the expected oncological and functional outcomes. One approach to improve treatment efficacy is to add novel molecular targeted agents to the classical treatment regimens (4).

Monoclonal antibodies targeting the epidermal growth factor receptor have shown clinical benefits in palliative and curative settings (5). However, only a minority of patients presenting with current or metastatic HNSCC exhibited tumor regression with these agents, and the majority of patients develop acquired tumor resistance following several months of treatments (4). Therefore in order to develop agents that target novel proteins, the identification of novel genes in HNSCC is required.

In the present study, the mutated genes in HNSCC were screened by searching the Cancer Genome Atlas (TCGA). The TCGA research network has profiled and analyzed large numbers of human tumors in order to identify molecular aberrations at DNA, RNA, protein and epigenetic levels (6-13). It was identified that there were many genes with amplification and deletion in HNSCC (Table I), including C9orf53. C9orf53 is a protein-coding gene. C9orf53 (also named CDKN2A-AS1) is associated with Alzheimer's disease (14). The present study focused on the role of $\mathrm{C} 9$ orf53 in HNSCC, and it was found that the main type of C9orf53 mutation in HNSCC is deletion. Importantly, it was observed that deletions in C9orf53 are associated with lower patient survival rates. In the cell experiments, the role of C9orf53 in HNSCC was investigated in vitro by overexpressing or inhibiting C9orf53 expression. In conclusion, the present study revealed the role of C9orf53 in HNSCC, and the study may provide a novel therapeutic target for future investigation.

\section{Materials and methods}

Frequency of gene alteration and patient survival analysis for cancer. The data on the frequency of C9orf53 alteration, mRNA level analysis and patient survival were obtained from 
TCGA via cBioportal for Cancer Genomics (http://www.cbioportal.org/public-portal/index.do) $(15,16)$.

Patients. Surgical specimens from 19 patients with HNSCC (age range: $36-75$ years; mean age: $54.3 \pm 7.4$ years; Sex ratio: Male to Female 1.7:1) and matched normal tissues adjacent to tumors were obtained postoperatively in June, 2009 from the Department of Head and Neck Surgery, Changhai Hospital, Second Military Medical University (Shanghai, China). All patients gave signed, informed consent for their tissues to be used for scientific research. Ethics approval for the present study was obtained from Changhai Hospital, Second Military Medical University (Shanghai, China). All diagnoses were based on pathological and/or cytological findings. The histological features of the specimens were evaluated by senior pathologists according to the World Health Organization classification criteria (17). The tissues were obtained prior to chemotherapy and radiotherapy. The samples were immediately frozen and stored at $-80^{\circ} \mathrm{C}$ prior to reverse transcription-quantitative polymerase chain reaction (RT-qPCR) assay.

Cell culture. 293, SCC-5 and SCC-9 cells were obtained from the Cell Bank of Chinese Academy of Science (Shanghai, China). 293, SCC-5 and SCC-9 cells were cultured in $37^{\circ} \mathrm{C}$, $5 \% \mathrm{CO}_{2}$, and in Dulbecco's modified Eagle's medium (Hyclone; GE Healthcare Life Sciences, Logan, UT, USA) supplemented with $10 \%$ fetal bovine serum (Hyclone; GE Healthcare Life Sciences), $2 \mathrm{mM}$ L-glutamine and $100 \mu \mathrm{g} / \mathrm{ml}$ penicillin/streptomycin (Sangon Biotech Co., Ltd., Shanghai, China) as described in previous studies (18-20).

RNA extraction and RT- $q P C R$. RNA was extracted with Trizol (Invitrogen; Thermo Fisher Scientific, Inc., Waltham, MA, USA) according to the manufacturer's protocol. The cDNA synthesis and RT-qPCR were subsequently performed using the Qiagen system as described previously (21). RT-qPCR analysis was performed using standard protocols on Applied Biosystem 7500 HT sequence Detection system. The relative mRNA levels of C9orf53 were normalized to levels of the housekeeping gene GAPDH and calculated using the $2^{-\Delta \Delta \mathrm{Cq}}$ method (22). The primers used are as follows: GAPDH forward, 5'-CCATGTTCGTCATGGG-TGTGAACCA-3' and reverse, 5'-GCCAGTAGAGGCAGGGATGATGTTG-3' and C9orf53 forward, 5'-AAGAATTCGGCACGAGGGTT-3' and reverse, 5'-CTCTGCCACAGTGGGATTGT-3'.

MTT assay. For MTT assay, 5x103 cells per well were seeded in triplicate in a 96-well plate with complete growth medium. The cells were counted over 5 days using the MTT assay (Promega Corporation, Madison, WI, USA) as described previously $(19,20)$. The data was measured using the Microtiter plate reader (Promega Corporation) at $570 \mathrm{~nm}$.

Transfection of C9orf53 overexpression plasmid and small-interfering (si)RNA. C9orf53 overexpression plasmid (pcDNA3.1-C9orf53;), C9orf53-siRNA (5'-GTGTGATTTCGTAAACAGATA-3') and control-siRNA were designed and constructed by Sangon Biotech Company (Shanghai, China). Untransfected cells were used as a blank control. Transfections were performed using Lipofectamine ${ }^{\circledR}$ 2000 (Invitrogen; Thermo Fisher Scientific, Inc.) according to the manufacturer's protocol. SCC-5 and SCC-9 cells were seeded into 24 -well plates at a density of $5 \times 10^{4}$ cells/well and were allowed to culture overnight. DNA Plasmid (500 ng) with Lipofectamine ${ }^{\circledR} 2000$ and siRNA $(600 \mathrm{ng})$ with Lipofectamine ${ }^{\circledR}$ 2000 were mixed for transfection. Then DNA-Lipofectamine ${ }^{\circledR}$ 2000 complexes were added into wells at $37^{\circ} \mathrm{C}$ for $24 \mathrm{~h}$ prior to subsequent experimentation.

Apoptosis assay. SCC-5 and SCC-9 cells were labeled with Annexin V-fluorescein isothiocyanate and propidium iodide (PI) using the apoptosis detecting kit (Invitrogen; Thermo Fisher Scientific, Inc.) following the manufacturer's instructions. Then samples were analyzed by fluorescence-activated cell sorting (FACS) assay with a flow cytometer (BD FACSVerse $^{\mathrm{TM}}$ flow cytometer, with BD FACSuite ${ }^{\mathrm{TM}}$ software v1.0.6, BD Biosciences, San Jose, CA, USA) (23).

Statistical analysis. Data are presented as the mean \pm standard deviation from at least three independent experiments. The difference between groups was analyzed using two-tailed, paired Student's t-test when only two groups were compared. The Wilcoxon matched-pairs signed rank test was used to determine if there was a statistically significant difference in the expression of C9orf53 between matched pairs. The difference between groups was analyzed using one-way ANOVA with post hoc contrasts by Student-Newman-Keuls test, when three or more than three groups were compared. Correlation analysis was performed using two-tailed Person's correlation coefficient analysis. Patient survival was determined by Kaplan-Meier analysis (with log-rank test). Statistical analyses were performed using SPSS software (version 17.0; IBM Corp., Armonk, NY, USA). P<0.05 was considered significantly different.

\section{Results}

Screening of novel genes amplified or deleted in HNSCC. To identify the potential target genes of HNSCC, the genes that are amplified or deleted in HNSCC were screened. It was observed that there were a number of genes amplified or deleted in HNSCC (Table I). To date, to the best of our knowledge, there is no study on the role of C9orf53 in HNSCC. Therefore, in the present study, the role of C9orf53 in HNSCC was investigated. Initially, mutations in C9orf53 were investigated in various types of cancer and mutations were identified in 302 HNSCC tissue samples. There were $27.5 \%$ HNSCC tissues with C9orf53 deletions (Fig. 1A). Next, it was observed that a low expression of C9orf53 mRNA was associated with C9orf53 deletions (Fig. 1B). Importantly, these data indicated that $\mathrm{C} 9$ orf53 deletion was associated with a decreased survival rate (Fig. 1C).

Reduced expression of C9orf53 in HNSCC tissues compared with normal tissues. Next, 19 surgical specimens and matched tumor-adjacent normal tissues were obtained from patients with HNSCC. The levels of C9orf53 mRNA were assayed by RT-qPCR. It was identified that 17 of the 19 HNSCC tissues exhibited lower C9orf53 expression compared with 
Table I. Expression of genes that are deleted or amplified in HNSCC.

\begin{tabular}{|c|c|}
\hline Genes & P-value \\
\hline \multicolumn{2}{|l|}{ Amplification } \\
\hline FADD & $\mathrm{P}<0.001$ \\
\hline USP13 & $\mathrm{P}<0.001$ \\
\hline DCUN1D1 & $\mathrm{P}<0.001$ \\
\hline EGFR & $\mathrm{P}<0.001$ \\
\hline CCND1 & $\mathrm{P}<0.001$ \\
\hline FGF3 & $\mathrm{P}<0.001$ \\
\hline MIR548K & $\mathrm{P}<0.001$ \\
\hline PPFIA1 & $\mathrm{P}<0.001$ \\
\hline POU5F1B & $\mathrm{P}<0.001$ \\
\hline CASC8 & $\mathrm{P}<0.001$ \\
\hline CCAT1 & $4.82 \times 10^{-22}$ \\
\hline FGFR1 & $1.15 \times 10^{-18}$ \\
\hline RGP1 & $1.24 \times 10^{-13}$ \\
\hline LINC00393 & $1.34 \times 10^{-11}$ \\
\hline MYRFL & $5.81 \times 10^{-11}$ \\
\hline MLANA & 0.147 \\
\hline CDK6 & 0.259 \\
\hline SAMD9L & 0.259 \\
\hline SNAI2 & $9.67 \times 10^{-9}$ \\
\hline NFIB & $2.38 \times 10^{-8}$ \\
\hline E2F1 & $9.36 \times 10^{-6}$ \\
\hline ANKRD39 & $5.09 \times 10^{-5}$ \\
\hline LRRC14B & $7.44 \times 10^{-5}$ \\
\hline ERBB2 & $1.16 \times 10^{-4}$ \\
\hline TINAG & $2.37 \times 10^{-4}$ \\
\hline CD44 & $5.72 \times 10^{-4}$ \\
\hline LINC00452 & $2.99 \times 10^{-3}$ \\
\hline PTP4A1 & 0.037 \\
\hline \multicolumn{2}{|l|}{ Deletion } \\
\hline C9ORF53 & $\mathrm{P}<0.001$ \\
\hline ZNF532 & $\mathrm{P}<0.001$ \\
\hline CDKN2A & $\mathrm{P}<0.001$ \\
\hline RN7SL5P & $1.42 \times 10^{-39}$ \\
\hline RNY3P4 & $3.13 \times 10^{-23}$ \\
\hline STK11 & $1.77 \times 10^{-19}$ \\
\hline FAM72C & $4.76 \times 10^{-19}$ \\
\hline PDE4D & $6.60 \times 10^{-18}$ \\
\hline RB1 & $4.14 \times 10^{-17}$ \\
\hline PARD3 & $1.80 \times 10^{-14}$ \\
\hline LINC00971 & 0.101 \\
\hline KIAA0825 & $1.25 \times 10^{-9}$ \\
\hline MIR3182 & $4.80 \times 10^{-9}$ \\
\hline CBWD3 & $2.74 \times 10^{-7}$ \\
\hline PTPRG & $1.29 \times 10^{-6}$ \\
\hline GPN2 & $1.62 \times 10^{-6}$ \\
\hline ZNF750 & $2.67 \times 10^{-6}$ \\
\hline KCNIP4 & $1.51 \times 10^{-5}$ \\
\hline RNA5SP431 & $1.61 \times 10^{-5}$ \\
\hline RAB24 & $1.09 \times 10^{-4}$ \\
\hline C9ORF163 & $1.24 \times 10^{-3}$ \\
\hline
\end{tabular}

Table I. Continued.

\begin{tabular}{lc}
\hline Genes & P-value \\
\hline LINC00645 & $7.31 \times 10^{-3}$ \\
LRRN1 & 0.0239 \\
\hline
\end{tabular}

The expression data were acquired from The Cancer Genome Atlas, where there were 302 HNSCC samples analyzed. The mean expression of these genes in tumor tissues and normal tissues were compared by paired t-test. HNSCC, Head and neck squamous cell carcinoma.

matched normal tissues that were adjacent to tumors (Fig. 2A). Furthermore, the median C9orf53 expression in HNSCC tissues was lower compared with normal tissues that were adjacent to tumors, and the standard deviation of C9orf53 expression in HNSCC was higher compared with normal tissues (Fig. 2B).

Reduced expression of C9orf53 promotes proliferation and overexpression of C9orf53-induced apoptosis. Next, the role of C9orf53 in vitro was investigated. The levels of C9orf53 in HNSCC cell lines (SCC-5 and SCC-9) were analyzed, and 293 cells were used as a control. It was observed that the levels of C9orf53 in SCC-5 and SCC-9 were lower compared with 293 cells (Fig. 3A). As SCC-5 cells exhibited lower C9orf53 levels compared with SCC-9 cells, C9orf53 was overexpressed in SCC-5 cells by transfection with pcDNA3.1-C9orf53. C9orf53 expression was suppressed in SCC-9 cells by transfection with si-C9orf53. The levels of C9orf53 were determined by RT-qPCR, 48 $\mathrm{h}$ following transfection. It was observed that the level of C9orf53 was successfully increased following transfection of pcDNA3.1-C9orf53 in SCC-5 cells and that C9orf53 expression was successfully inhibited following transfection with si-C9orf53 in SCC-9 cells (Fig. 3B). As transfection with si-C9orf53-1 resulted in the greatest decrease in C9orf53 expression (Fig. 3B), si-C9orf53-1 was selected for subsequent experiments. Following the overexpression or inhibition of C9orf53, proliferation was examined by MTT assay. It was found that overexpressing C9orf53 inhibited proliferation, and suppressing $\mathrm{C} 9$ orf53 promoted proliferation (Fig. 3C). Next, the cell apoptosis was examined by FACS assay. The overexpression of C9orf53 increased the rate of cell apoptosis (Fig. 3D).

\section{Discussion}

In the present study, the role of C9orf53 in HNSCC was studied. It was identified that $\mathrm{C} 9$ orf53 was deleted in HNSCC, and the levels of C9orf53 in HNSCC tissues were lower in tumor tissues compared with matched normal tissues that were adjacent to tumor tissues, Notably, the deletion of C9orf53 and a lower expression level of C9orf53 were associated with the rate of patient survival. Subsequently, the role of C9orf53 was confirmed by overexpressing and suppressing C9orf53 expression in vitro. The suppression of C9orf53 was able to promote proliferation 
A

C9orf53 deletion

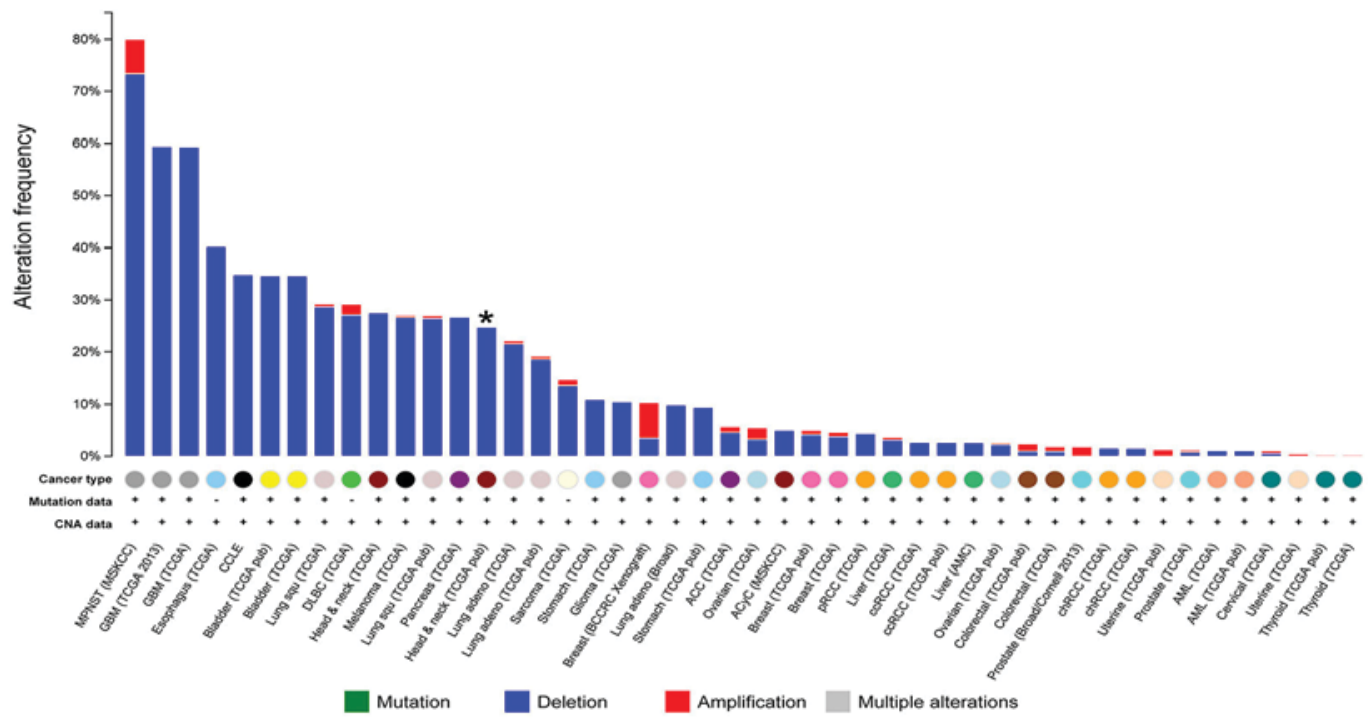

B

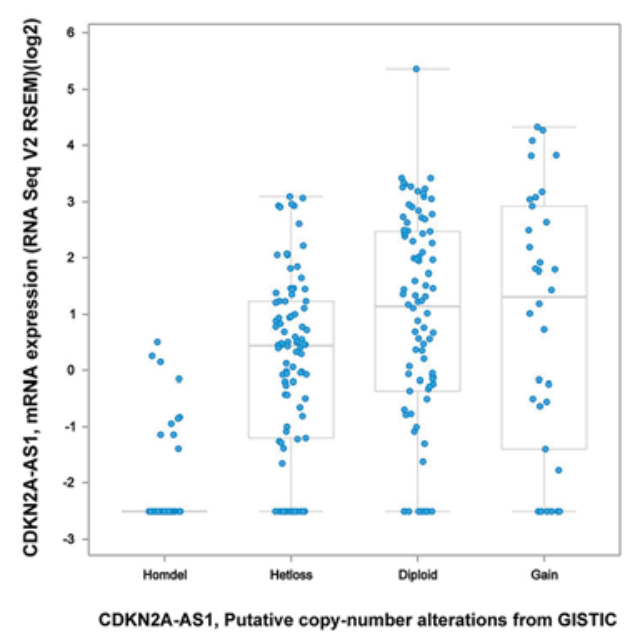

C

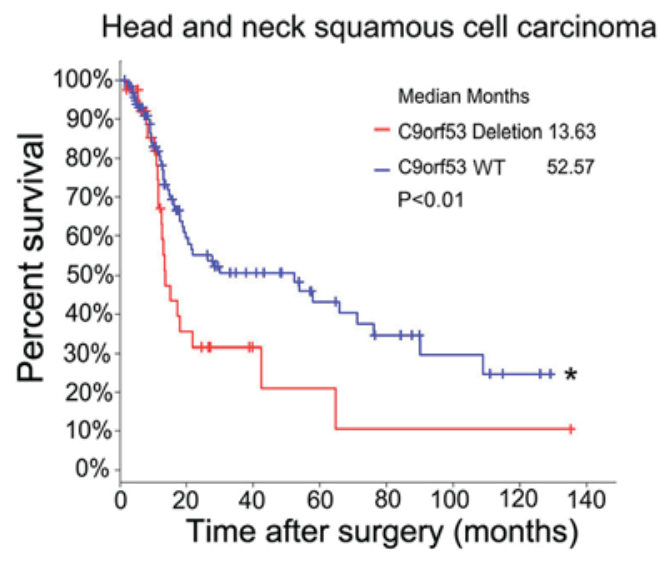

Figure 1. Low C9orf53 level in HNSCC tissues is associated with low survival rates. Data on the alteration frequency of C9orf53 and mRNA levels were obtained from TCGA via cBioportal for Cancer Genomics (http://www.cbioportal.org/public-portal/index.do). (A) Alterations in C9orf53 were visualized by cBioPortal for Cancer Genomics. Mutation, deletion, amplification and multiple alterations are shown in different colors. "Indicating head and neck tumor. (B) Comparison of C9orf53 mRNA level with the copy number of C9orf53 in HNSCC. (C) C9orf53 deletion was correlated with lower patient survival rates. The median months of survival for patients with C9orf53 deletion mutation was 13.63 months. ${ }^{*} \mathrm{P}<0.05$. C9ORF53, CDKN2A antisense RNA 1; HNSCC, Head and neck squamous cell carcinoma; homdel, homozygous deletion; hetloss, heterozygous loss; TCGA, The Cancer Genome Atlas; WT, wild type.

and induce apoptosis and apoptosis. To the best of our best knowledge, this might be the first report of the role of C9orf53 in cancer.

The data from the present study indicated that the effects of C9orf53 on proliferation and apoptosis were relative minor. However, the difference in survival between patients with C9orf53 deletion and wild-type C9orf53 was significant. It is surprising that these minor influences were able to cause an effect on patient survival. It was hypothesized that although the effects of C9orf53 on cell proliferation and apoptosis were rather minor, the cells proliferated exponentially in vivo. Therefore, minor differences in C9orf53 were able to cause a long-lasting effect on patient survival (130 months).
Recently, a comprehensive landscape of somatic genomic alteration of HNSCC was provided by TCGA (24). It was indicated that human-papillomavirus-associated tumors are dominated by helical domain mutations of the oncogene phosphatidylinositol-4,5-bisphosphate 3-kinase catalytic subunit $\alpha$. Smoking-related HNSCCs demonstrated near universal loss-of-function tumor protein p53 mutations and CDKN2A inactivation. Whether the role of C9orf53 in HNSCC is associated with human papillomavirus infection or smoking will be investigated in further studies.

In conclusion, the present study investigated the role of C9orf53 in HNSCC. The data indicated that the deletion 

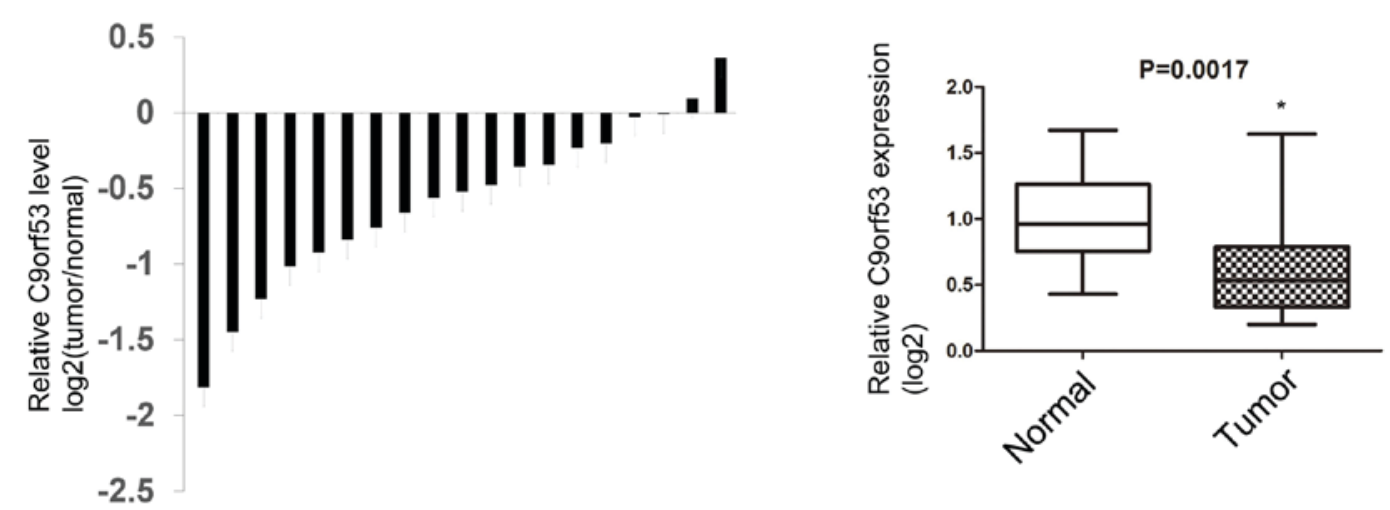

Figure 2. C9orf53 expression in HNSCC tissues is lower compared with normal tissues. The levels of C9orf53 in 19 surgical specimens of patients with HNSCC were assayed by RT-qPCR. The data are expressed in the form of $\log 2$ (tumor/normal). (A) The levels of C9orf53 in 19 surgical specimens and in matched tumor-adjacent normal tissues. (B) All RT-qPCR experiments were performed in triplicate, and results are displayed in a box-plot. The difference between the groups was analyzed using t-test. " $\mathrm{P}<0.05$. C9ORF53, CDKN2A antisense RNA 1; RT-qPCR, reverse transcription-quantitative polymerase chain reaction.

A

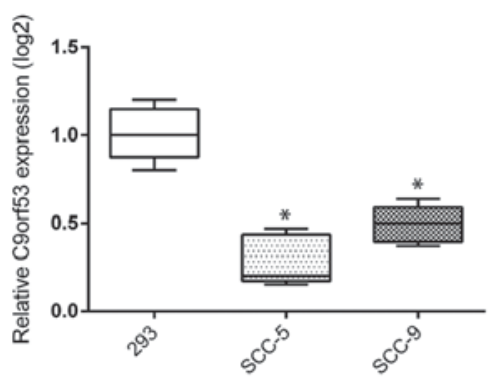

C

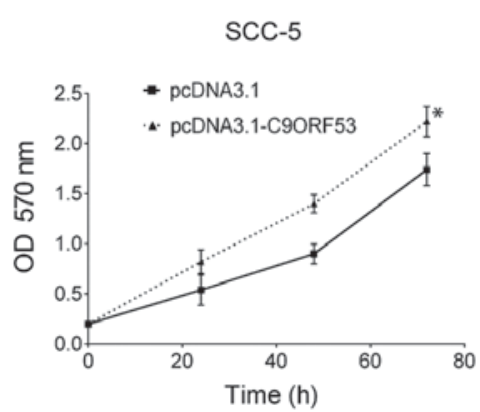

$\mathrm{B}$

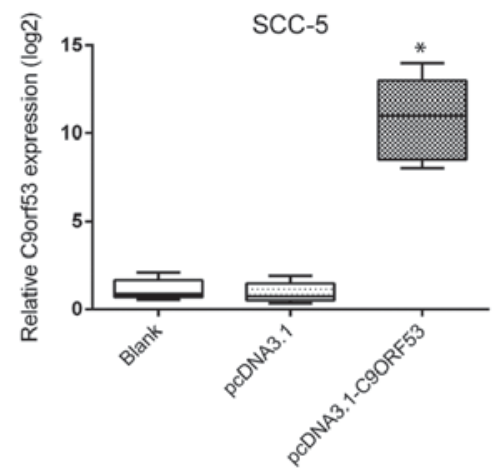

SCC-9

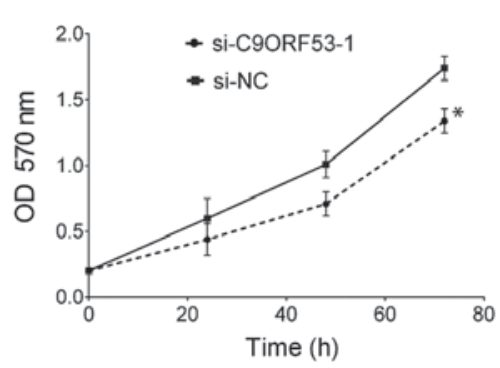

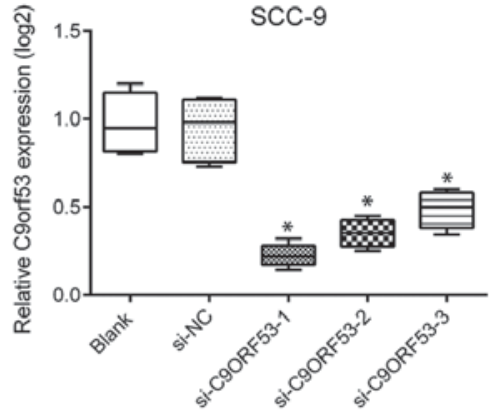

D

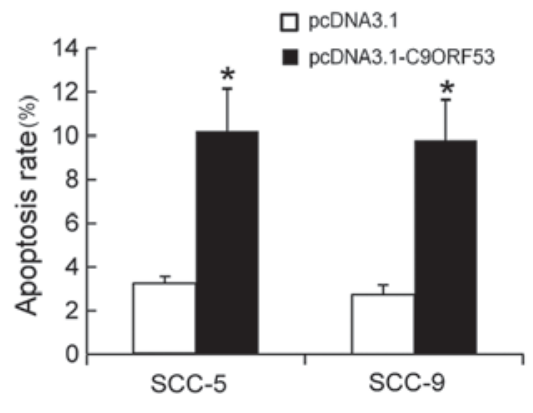

Figure 3. Suppression of C9orf53 promotes proliferation and induces apoptosis. The levels of C9orf53 in 293, SCC-5 and SCC-9 cells were assayed by RT-qPCR (A) Data on C9orf53 expression are displayed in box plots. "P<0.05 vs. 293 cells. (B) The levels of C9orf53 in SCC-5 cells were assayed by RT-qPCR $48 \mathrm{~h}$ following transfection of pcDNA3.1-C9orf53. All data on C9orf53 expression are displayed in box plots. "P<0.05 pcDNA3.1-C9orf53 vs. control. (C) Following the transfection of pcDNA3.1-C9orf53 or siC9orf53-1, cell proliferation was analyzed by MTT assay at the indicated time points. (D) Cell apoptosis was analyzed by FACS, $48 \mathrm{~h}$ following the transfection of pcDNA3.1-C9orf53 or siC9orf53-1. All experiments were repeated four times. The differences between two groups were analyzed using two-tailed Student's t-test, and the differences between groups were analyzed using one-way ANOVA when three or more groups were compared. " $\mathrm{P}<0.05$ vs. pcDNA3.1-transfected cells. C9ORF53, CDKN2A antisense RNA 1; FACS, fluorescence-activated cell sorting analysis; $\mathrm{NC}$, negative control; OD, optical density; RT-qPCR, reverse transcription-quantitative polymerase chain reaction; siRNA, small-interfering RNA.

and decreased level of C9orf53 might promote the growth of HNSCC cells. The present study might provide a novel therapeutic target for further investigations.

\section{Acknowledgements}

The authors would like to thank Dr. Chaoxiong Zhang (West China School of Public Health and Healthy Food Evaluation
Research Center, Sichuan University, Chengdu, China) for assistance with the discussion.

\section{Funding}

The present study was supported by the National Natural Science Foundation of China (grant no. 81271353) and PLA Research Project (grant no. 2011XL015) and 'The 12th 
Five-Year Plan' for Medicsal Science Development of PLA Research Project (grant no. CWS11J300), the Key Project on the Integration of Industry, Education, and Research and Medicine of Science and Technology Commission of Shanghai Municipality (grant nos. 12DZ1940503 and 13DZ1942704).

\section{Availability of data and materials}

All data generated or analyzed during the present study are included in this published article.

\section{Authors' contributions}

GW, HJ and YL collected patient data and performed cell experiments. YW and XC performed transfection and apoptosis analysis. YZ and DW contributed to study design and manuscript writing.

\section{Ethics approval and consent to participate}

The present study was approved by Ethics Committee of Second Military Medical University (Shanghai, China).

\section{Patient consent for publication}

All patients gave informed consent for the use of their tissues and publication of the data.

\section{Competing interests}

The authors declare that they have no competing interests.

\section{References}

1. Liu CJ, Lin SC, Chen YJ, Chang KM and Chang KW: Array-comparative genomic hybridization to detect genomewide changes in microdissected primary and metastatic oral squamous cell carcinomas. Mol Carcinog 45: 721-731, 2006.

2. Shieh TM, Lin SC, Liu CJ, Chang SS, Ku TH and Chang KW: Association of expression aberrances and genetic polymorphisms of lysyl oxidase with areca-associated oral tumorigenesis. Clin Cancer Res 13: 4378-4385, 2007.

3. Ferlay J, Shin HR, Bray F, Forman D, Mathers C and Parkin DM: Estimates of worldwide burden of cancer in 2008: GLOBOCAN 2008. Int J Cancer 127: 2893-2917, 2010.

4. Schmitz S, Ang KK, Vermorken J, Haddad R, Suarez C, Wolf GT, Hamoir M and Machiels JP: Targeted therapies for squamous cell carcinoma of the head and neck: Current knowledge and future directions. Cancer Treat Rev 40: 390-404, 2014.

5. Allegra CJ, Jessup JM, Somerfield MR, Hamilton SR, Hammond EH, Hayes DF, McAllister PK, Morton RF and Schilsky RL: American society of clinical oncology provisional clinical opinion: Testing for KRAS gene mutations in patients with metastatic colorectal carcinoma to predict response to anti-epidermal growth factor receptor monoclonal antibody therapy. J Clin Oncol 27: 2091-2096, 2009.
6. Cancer Genome Atlas Research Network, Weinstein JN, Collisson EA, Mills GB, Shaw KR, Ozenberger BA, Ellrott K, Shmulevich I, Sander C and Stuart JM: The cancer genome atlas pan-cancer analysis project. Nat Genet 45: 1113-1120, 2013

7. Verhaak RG, Hoadley KA, Purdom E, Wang V, Qi Y Wilkerson MD, Miller CR, Ding L, Golub T, Mesirov JP, et al: Integrated genomic analysis identifies clinically relevant subtypes of glioblastoma characterized by abnormalities in PDGFRA, IDH1, EGFR, and NF1. Cancer Cell 17: 98-110, 2010.

8. Cancer Genome Atlas Research Network: Integrated genomic analyses of ovarian carcinoma. Nature 474: 609-615, 2011.

9. Cancer Genome Atlas Research Network: Comprehensive genomic characterization of squamous cell lung cancers. Nature 489: 519-525, 2012.

10. Cancer Genome Atlas Network: Comprehensive molecular characterization of human colon and rectal cancer. Nature 487: 330-337, 2012.

11. Forbes SA, Bindal N, Bamford S, Cole C, Kok CY, Beare D, Jia M, Shepherd R, Leung K, Menzies A, et al: COSMIC: Mining complete cancer genomes in the catalogue of somatic mutations in cancer. Nucleic Acids Res 29: D945-D950, 2011.

12. Collins FS and Barker AD: Mapping the cancer genome. Scientific American 296: 50-57, 2007.

13. Stratton MR, Campbell PJ and Futreal PA: The cancer genome. Nature 458: 719-724, 2009.

14. Emanuele E, Lista S, Ghidoni R, Binetti G, Cereda C, Benussi L, Maletta R, Bruni AC and Politi P: Chromosome 9p21.3 genotype is associated with vascular dementia and Alzheimer's disease. Neurobiol Aging 32: 1231-1235, 2011.

15. Cerami E, Gao J, Dogrusoz U, Gross BE, Sumer SO, Aksoy BA, Jacobsen A, Byrne CJ, Heuer ML, Larsson E, et al: The cBio cancer genomics portal: an open platform for exploring multidimensional cancer genomics data. Cancer Discov 2: 401-404, 2012.

16. Gao J, Aksoy BA, Dogrusoz U, Dresdner G, Gross B, Sumer SO, Sun Y, Jacobsen A, Sinha R, Larsson E, et al: Integrative analysis of complex cancer genomics and clinical profiles using the cBioPortal. Sci Signal 6: pl1, 2013.

17. Barnes L, Eveson JW, Reichart P and Sidransky D: Pathology and genetics of head and neck tumours. IARC, 2005.

18. Wu N, Liu C, Bai C, Han YP, Cho WC and Li Q: Over-expression of deubiquitinating enzyme USP14 in lung adenocarcinoma promotes proliferation through the accumulation of beta-catenin. Int J Mol Sci 14: 10749-10760, 2013.

19. Wu N, Zhang C, Bai C, Han YP and Li Q: miR-4782-3p Inhibited non-small cell lung cancer growth via USP14. Cell Physiol Biochem 33: 457-467, 2014.

20. Song B, Zhang C, Li G, Jin G and Liu C: MiR-940 inhibited pancreatic ductal adenocarcinoma growth by targeting MyD88. Cell Physiol Biochem 35: 1167-1177, 2015.

21. Hou J, Lin L, Zhou W, Wang Z, Ding G, Dong Q, Qin L, Wu X, Zheng Y, Yang Y, et al: Identification of miRNomes in human liver and hepatocellular carcinoma reveals miR-199a/b-3p as therapeutic target for hepatocellular carcinoma. Cancer Cell 19: 232-243, 2011.

22. Livak KJ and Schmittgen TD: Analysis of relative gene expression data using real-time quantitative PCR and the 2- $\Delta \Delta \mathrm{CT}$ method. Methods 25: 402-408, 2001.

23. Lu J, Wen M, Huang Y, He X, Wang Y, Wu Q, Li Z, Castellanos-Martin A, Abad M, Cruz-Hernandez JJ, et al: C2ORF40 suppresses breast cancer cell proliferation and invasion through modulating expression of $\mathrm{M}$ phase cell cycle genes. Epigenetics 8: 571-583, 2013.

24. Cancer Genome Atlas Network: Comprehensive genomic characterization of head and neck squamous cell carcinomas. Nature 517: 576-582, 2015. 